\title{
Chairman's Summing Up
}

Professor J. R. Learmonth (Department of Surgery, University New Buildings, Edinburgh): I have sat here throughout the day and have been most profoundly impressed by all the work done on nutritional factors in the healing of surgical conditions, and I cannot imagine any sceptical surgeon who would not have been convinced of the importance of the work. In the future, this work must be brought into much closer contact with those doing clinical work in hospitals.

First of all, there is the desirability that patients should arrive at hospital for an operation or attention to a fracture in a state of nutrition which would relieve surgeons of the responsibility for correcting deficiencies; this is a matter of national planning. At the moment, we are chiefly concerned with those whose assimilation of food has been altered in some way, for example patients with cancer of the pylorus, and in the future, one would hope that these people would be diagnosed so early that when they come to hospital for treatment any nutritional adjustment required. would be minimal. There should be a wider pooling of cases with clinical conditions, in which those interested might be of great assistance by transferring to centres, where the work could be done, patients suffering from lesions, such as short circuits in the alimentary canal, likely to repay biochemical investigation.

That raises a point which $\mathrm{Mr}$. Croft brought up as to the availability of the different amino-acids in a mixture; a study of one or two such patients would be profoundly interesting to clinicians. I am sure any such cases could be made available.

I understand that this Society contains a goodly number of dieticians. The problems of the moment for them in hospital are to accumulate sufficient food and accessory food factors, and to present them in a palatable form. As I go round the wards I see patients who have a tube in every natural orifice and one or two who have tubes in unnatural orifices; I do not think this is conducive to recovery from an operation. I put it to nutritionists that there is a psychological side too.

One of the problems which this Society should take up is to induce hospitals to present an adequate and balanced diet in a form which is palatable to the patients and which is particularly adapted to the locality from which they come. I cannot myself believe that a glutinous mess, which must be food only because it is on a food tray, will do much to improve the condition from which a patient is suffering, even though it may prove on analysis to be biochemically adequate.

One other point occurs to me; many of the discussions today have been about the rate at which epithelium will cover a raw surface. The modern surgical trend is to apply a skin graft to all but the smallest raw surface, and it might be worth while studying the effect of various deficiencies on the percentage takes in different types of skin graft. 